\title{
The importance of gray color as a dermoscopic clue in facial pigmented lesion evaluation: a case report
}

\author{
Danica Tiodorovic-Zivkovic ${ }^{1}$, Iris Zalaudek ${ }^{2,3}$, Aimilios Lallas ${ }^{2}$, Alexander J. Stratigos ${ }^{4}$, \\ Simonetta Piana ${ }^{5}$ Giuseppe Argenziano ${ }^{2}$
}

\begin{abstract}
1 Clinic of Dermatovenerology, Clinical Center of Nis, Medical Faculty, University of Nis, Serbia
2 Skin Cancer Unit, Arcispedale Santa Maria Nuova, IRCCS, Reggio Emilia, Italy

3 Department of Dermatology, Medical University of Graz, Graz, Austria

4 Dermato-Oncology Unit, Department of Dermatology, University of Athens Medical School, Andreas Sygros Hospital, Athens, Greece

5 Unit of Anatomic Pathology, Arcispedale Santa Maria Nuova IRCCS, Reggio Emilia, Italy
\end{abstract}

Key words: melanoma, lentigo maligna, solar lentigo, dermoscopy

Citation: Tiodorovic-Zivkovic D, Zalaudek I, Lallas A, Stratigos AJ, Piana S, Argenziano G. The importance of gray color as a dermoscopic clue in facial pigmented lesion evaluation: a case report. Dermatol Pract Conc. 2013;3(4):9. http://dx.doi.org/10.5826/dpc.0304a09.

Received: May 20, 2013; Accepted: July 17, 2013; Published: October 31, 2013

Copyright: @2013 Tiodorovic-Zivkovic et al. This is an open-access article distributed under the terms of the Creative Commons Attribution License, which permits unrestricted use, distribution, and reproduction in any medium, provided the original author and source are credited.

Funding: Study supported in part by the Italian Ministry of Health (RF-2010-2316524).

Competing interests: The authors have no conflicts of interest to disclose.

All authors have contributed significantly to this publication.

Corresponding author: Giuseppe Argenziano, M.D., Skin Cancer Unit, Arcispedale Santa Maria Nuova IRCCS, Viale Risorgimento 80—42100 Reggio Emilia, Italy. Tel. +39 335 415093; Fax. E-mail: g.argenziano@gmail.com

\section{Clinical findings}

A 57-year-old man presented with a solitary flat pigmented lesion on his face, which had appeared two years previously and was slowly enlarging over time. Physical examination revealed a flat pigmented lesion, $8 \mathrm{~mm}$ in size, on the patient's right cheek. The clinical diagnosis was suggestive of a solar lentigo (SL) because of the symmetric shape, the regular border, and the homogenous brown color (Figure 1). Pigmented actinic keratosis (PAK) and lentigo maligna (LM) were also included in the differential diagnosis.

Dermoscopically, the lesion exhibited a brown pseudonetwork and a striking gray color, especially in the center (Figure 2). There were large gray circles that resembled rhomboidal structures, though unconvincingly, in the absence of the well-known clues for the diagnosis of LM. However, the presence of gray color, asymmetrically distrib-

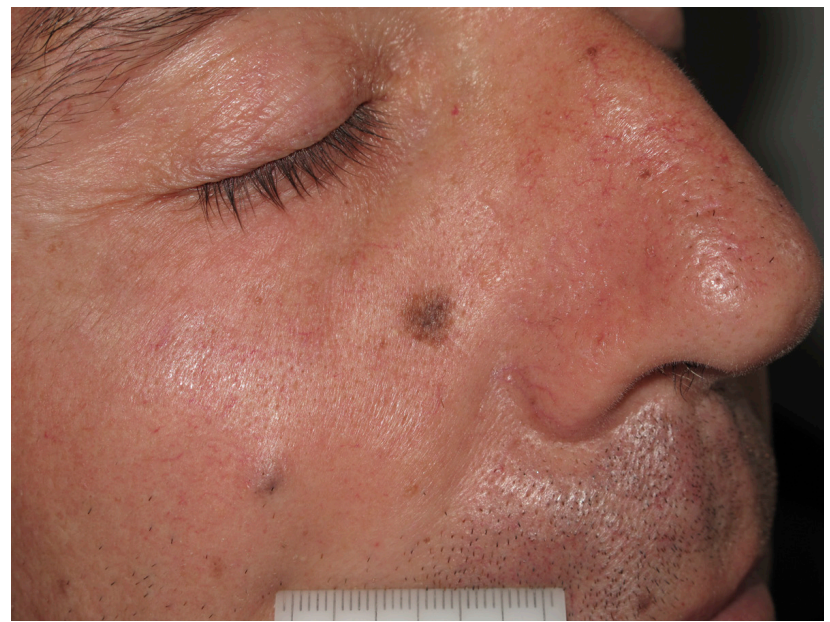

Figure 1. Clinical image of solitary flat pigmented lesion on the face. [Copyright: @2013 Tiodorovic-Zivkovic et al.] 


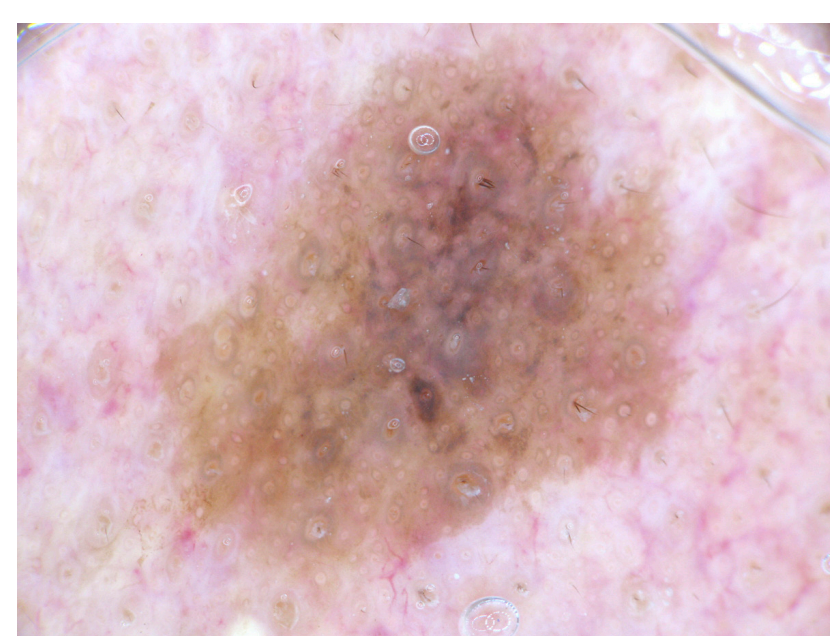

Figure 2. Dermoscopic examination of the lesion showed striking gray color. [Copyright: (92013 Tiodorovic-Zivkovic et al.]

uted, prompted us to perform a punch biopsy and a histopathologic examination.

\section{Histopathological findings}

Histopathologically, the lesion was characterized by a junctional proliferation of atypical melanocytes with pleomorphic nuclei, as solitary units that displayed crowding and uneven distribution, along with presence of junctional nests of melanocytes. In addition, melanocytes were seen in pagetoid spread. Marked solar elastosis was present within the underlying dermis (Figure 3).

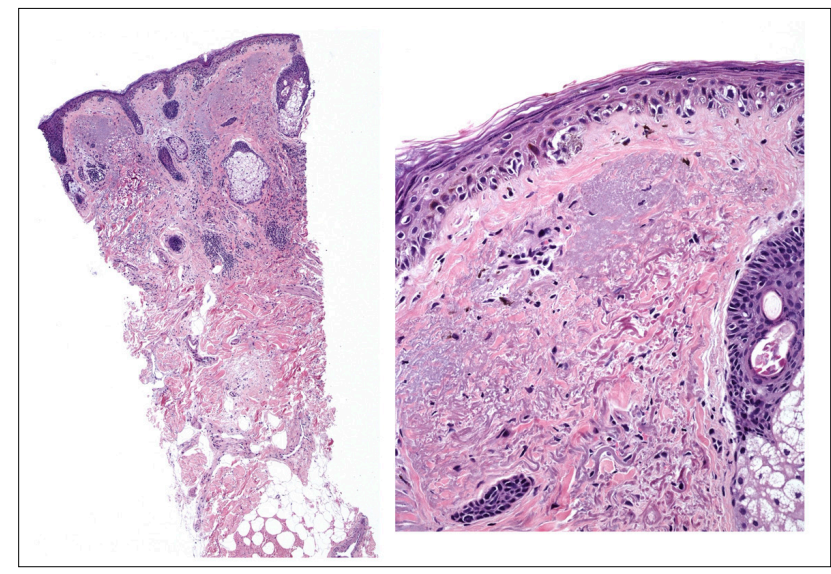

Figure 3. Histopathologic examination revealed an early lentigo maligna. [Copyright: (C2013 Tiodorovic-Zivkovic et al.]

\section{Diagnosis}

Melanoma in situ (lentigo maligna type) on chronically sun damaged skin.

\section{Discussion}

The differential diagnosis of flat pigmented macules on chronically sun-damaged facial skin is challenging and includes SL or early seborrheic keratosis (SK), PAK, LM and lichen planus-like keratosis (LPLK) [1]. Especially in the context of early lesions, clinical characteristics can be insufficient to allow differentiation among these entities, since they all present as flat pigmented lesions typically developing on sundamaged skin of elderly individuals. In contrast to their clinical similarity, these entities significantly differ in their physical course, prognosis and management strategies. Accordingly, accurate discrimination among them is mandatory.

Dermoscopy improves the diagnostic accuracy when evaluating pigmented skin lesions and is nowadays considered an irreplaceable part of clinical examination of skin tumors [2]. However, evaluation of pigmented facial lesions remains challenging, even with the addition of dermoscopic information $[3,4]$.

The traditional dermoscopic criteria of LM include asymmetrically pigmented follicles, circle within the circle, annulargranular pattern, rhomboidal structures, obliteration of the hair follicles and gray pseudo-network [5,6]. Darkening at dermoscopic examination, target-like pattern, red rhomboidal structures and increased density of the vascular network are recently described additional features [7]. Dermoscopy of a large-size LM typically reveals several of the above criteria, allowing a straightforward diagnosis. However, at earlier stages, such as the LM presented herein, dermoscopic criteria of LM have not yet fully developed. In such cases, clinical differentiation from benign pigmented lesions remains troublesome even when coupled with dermoscopy. This is because each of the classic LM criteria may also be present in SL/early SK and PAK, and only the simultaneous presence of four or more criteria has been shown to predict accurately the diagnosis of melanoma.

Interestingly, all the classic LM criteria have a common denominator, namely, the gray color. The example of the patient presented here highlights that grey color can be detected even before the formation of the characteristic LM structures, such as circles or rhomboids. In our estimation, this is the single most sensitive feature for the dermoscopic recognition of early facial melanoma and its presence should always prompt the clinician to perform a biopsy.

\section{Learning points}

- Clinical differentiation of early LM from solar lentigo/ early seborrheic keratosis and pigmented actinic keratosis is challenging. Dermoscopy may enhance the clinical diagnosis, but in small sized LM the typical diagnostic criteria could be not yet fully developed.

- In our estimation, gray color is the single most sensitive feature for the dermoscopic recognition of facial melanoma and its presence should always prompt the clinician to perform a biopsy. 


\section{References}

1. Akay BN, Kocyigit P, Heper AO, Erdem C. Dermatoscopy of flat pigmented facial lesions: diagnostic challenge between pigmented actinic keratosis and lentigo maligna. Br J Dermatol. 2010;163(6):1212-7.

2. Rosendahl C, Tschandl P, Cameron A, Kittler H. Diagnostic accuracy of dermatoscopy for melanocytic and nonmelanocytic pigmented lesions. J Am Acad Dermatol. 2011;64(6):1068-73.

3. Stante M, Giorgi V, Stanganelli I, Alfaioli B, Carli P. Dermoscopy for early detection of facial lentigo maligna. Br J Dermatol. 2005;152(2):361-4.
4. Schiffner R, Perusquia AM, Stolz W. One-year follow-up of a lentigo maligna: first dermoscopic signs of growth. Br J Dermatol. 2004;151(5):1087-9.

5. Stolz W, Schiffner R, Burgdorf WH. Dermatoscopy for facial pigmented skin lesions. Clin Dermatol. 2002;20(3):276-8.

6. Tanaka M, Sawada M, Kobayashi K. Key points in dermoscopic differentiation between lentigo maligna and solar lentigo. J Dermatol. 2011;38(1):53-8.

7. Pralong P, Bathelier E, Dalle S, et al. Dermoscopy of lentigo maligna melanoma: report of 125 cases. Br J Dermatol. 2012;167(2):280-7. 